\title{
riccafd
}

Revista Iberoamericana de Ciencias de la Actividad Física y el Deporte

\section{IMPORTANCIA DE UNA HERRAMIENTA TECNOLÓGICA EN LA GESTIÓN DE INFORMACIÓN EN EL DEPORTE. PERCEPCIÓN DEL STAFF TÉCNICO DE UN EQUIPO DE VOLEIBOL DE ALTO NIVEL}

IMPORTANCE OF A TECHNOLOGICAL TOOL IN INFORMATION MANAGEMENT IN SPORTS. PERCEPTION OF THE TECHNICAL STAFF OF A HIGH LEVEL VOLLEYBALL TEAM

Fernández-Echeverría, C. ${ }^{1}$, Mesquita, I. ${ }^{2}$, Conejero, M. ${ }^{3}$ y Moreno, M.P. ${ }^{4}$

${ }^{1}$ Fernández-Echeverría, C.Personal Contratado Investigador de la de la Universidad de Extremadura. Becaria predoctoral de la Fundación Tatiana Pérez de Guzmán el Bueno. Facultad de Ciencias del Deporte de Cáceres (España).cafernandeze@unex.es

2 Mesquita, I. Profesora Asociada con Agregación de la Universidad de Oporto. Faculdade de Desporto de Porto (Portugal). imesquita@fade.up.pt

${ }^{3}$ Conejero, M. Personal Contratado Investigador de la de la Universidad de Extremadura. Becario predoctoral de la Fundación Fernando Valhondo. Facultad de Ciencias del Deporte de Cáceres (España). mconejerx@alumnos.unex.es

${ }^{3}$ Moreno, M.P. Profesora Titular de la Universidad de Extremadura. Facultad de Ciencias del Deporte de Cáceres (España).pmoreno@unex.es

\section{AGRADECIMIENTOS}

Este trabajo ha sido desarrollado gracias a financiación otorgada por la fundación Tatiana Pérez de Guzmán el Bueno y gracias a la aportación de la Consejería de Economía e Infraestructuras de la Junta de Extremadura a través del Fondo Europeo de Desarrollo Regional.

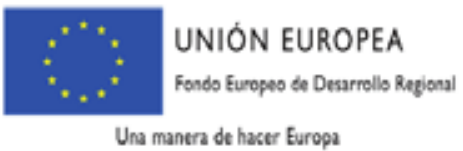

Una manera de hacer Europa

\section{JUNTA DE EXTREMADURA}

Consejeria de Economia e infraestructura

Código UNESCO: 5899 Otras Especialidades Pedagógicas (Educación Física y Deporte) Clasificación Consejo de Europa: 16. Sociología del deporte DOI:http://dx.doi.org/10.24310/riccafd.2018.v7i3.5541

\section{RESUMEN}

El objetivo del presente estudio fue conocer la percepción del staff técnico de un equipo de voleibol de alto nivel de la viabilidad, contextualización y utilidad, de una herramienta tecnológica, con posibilidad de acceso desde dispositivos 
móviles, empleada para almacenar e intercambiar información. La muestra estuvo formada por el staff técnico de un equipo de voleibol femenino que compite en alto nivel, compuesto por un entrenador y un entrenador asistente con funciones de preparador físico. El uso de la herramienta tecnológica, por el equipo, se realizó durante una temporada (siete meses). La herramienta tecnológica consta de una página web y su aplicación con acceso directo a la información desde dispositivos móviles. Como técnica de recogida de datos, se empleó la entrevista semiestructurada al terminar la temporada. El análisis de los datos se realizó a través de la Grounded Theory. Nuestros resultados indican que el staff técnico considera dicha herramienta tecnológica como viable, contextualizada y útil, permitiendo organizar, estructurar e intercambiar información de forma sencilla. Además, los resultados destacan que la posibilidad de acceso mediante un dispositivo móvil tiene grandes ventajas (acceso desde cualquier lugar y en cualquier momento), y alguna desventaja como la posible pérdida de contacto humano.

Palabras clave: nuevas tecnologías, entrenador, voleibol, gestión de información, alto nivel.

\section{ABSTRACT}

The objective of the present study was to determine the perception of the technical staff of a high level volleyball team about the viability, contextualization and utility of a technological tool, available in mobile devices, used to store and exchange information. The sample was formed by the technical staff of a female volleyball team that competes in a high level league, composed of a trainer and an assistant trainer with functions of physical trainer. The use of the technological tool by the team was carried out during a complete season (7 months). The technological tool consists of a web page and its application with direct access to information through mobile devices. Data collection was carried out from semi-structured interviews applied in the end of the season. Data analysis was performed through the Grounded Theory. Our results indicate that the technical staff considers this technological tool viable, contextualized and useful, allowing to organize structure and exchange information in a simple way. In addition, the results show that the possibility of access from mobile devices provides great advantages (permanent access to information and from anywhere) and some disadvantage such as the possible loss of human contact.

Keywords: new technologies, coach, volleyball, information management, high level.

\section{INTRODUCCIÓN}

El desarrollo de las nuevas tecnologías no ha pasado desapercibido en el ámbito de las Ciencias del Deporte ${ }^{1}$. Dicha incorporación comenzó con la utilización 
de aplicaciones informáticas vinculadas a la investigación del comportamiento humano, fundamentalmente en fisiología, biomecánica y psicología ${ }^{2}$. Sin embargo, rápidamente se introdujeron en el ámbito del entrenamiento deportivo ${ }^{1,3}$, con el objetivo de facilitar la labor de los técnicos e incrementar el rendimiento de los deportistas ${ }^{4-6}$, convirtiéndose éstas en herramientas indispensables para realizar eficazmente un proceso de planificación, control y análisis, tanto técnico como táctico, del entrenamiento deportivo ${ }^{7}$.

Dichas herramientas tecnológicas, para que puedan ser introducidas de forma sencilla en los entrenamientos o rutinas deportivas, deben de ser útiles y de fácil utilización, ya que si son muy complejas y no ayudan o entorpecen su labor diaria, no las incorporaran como herramientas de trabajo ${ }^{1}$.

En este sentido, las herramientas tecnológicas que ayudan a la labor diaria de los técnicos y deportistas, podemos dividirlas en software que facilitan el análisis estadístico y estratégico del entrenamiento y la competición ${ }^{8,9}$, y software que facilitan la planificación y el control del entrenamiento ${ }^{10,11}$. Dichos software pueden ofrecer información a nivel físico ${ }^{12}$, biomecánico ${ }^{13}$, técnico ${ }^{14}$ o táctico ${ }^{15,16}$, entre otras.

Además, la evolución de las nuevas tecnologías va a velocidad vertiginosa ${ }^{6}$. En este sentido, con el objetivo de poder utilizar las nuevas tecnologías en cualquier momento y desde cualquier lugar, las herramientas tecnológicas han pasado de ser sistemas fijos a sistemas móviles ${ }^{4,17}$, llegando así, a una mayor cantidad de usuarios ${ }^{18}$.

Este hecho ha beneficiado la incorporación de las nuevas tecnologías, aún más si cabe, en el ámbito deportivo, ya que existen aplicaciones para dispositivos móviles de fácil uso durante el entrenamiento deportivo, como pueden ser aquellas aplicaciones que ayudan a crear programas de acondicionamiento físico con imágenes y videos explicativos ${ }^{19}$, o aquellas que permiten medir de forma rápida y sencilla, mediante un dispositivo móvil, el estado fisiológico del deportista $^{20}$ o medir el salto ${ }^{21}$.

En este sentido, algunos estudios se han preocupado por conocer la percepción de los usuarios sobre la utilidad de dichas herramientas tecnológicas, obteniendo una percepción positiva sobre las mismas ${ }^{22,10}$. En nuestro caso, una de estas actividades en la que la tecnología puede ayudar al staff técnico es en la gestión e intercambio de información con sus equipos, debido a la gran cantidad de información que se maneja, en muchas ocasiones de forma poco eficiente y sistematizada. Además, si dicha herramienta puede ser utilizada desde un dispositivo móvil, facilitará en gran medida la gestión de esa información. Por ello, el objetivo del presente estudio es conocer la percepción del staff técnico de un equipo de voleibol de alto nivel de la viabilidad, contextualización y utilidad, de una herramienta tecnológica, con posibilidad de acceso desde dispositivos móviles, empleada para almacenar e intercambiar información. 


\section{MÉTODO}

\section{Participantes}

La muestra del estudio está formada por el staff técnico de un equipo de voleibol femenino que compite en alto nivel. Dicho staff técnico está compuesto por un primer entrenador (entrenador nivel III, con una experiencia de 25 años en el entrenamiento y competición en el alto nivel y en etapas de formación) y un entrenador asistente con labores de preparador físico (entrenador nivel III, con una experiencia de 6 años en el entrenamiento y en la preparación física en el alto nivel).

Se garantizó a los participantes en el estudio la confidencialidad y el anonimato durante todo el proceso, incluido el registro de datos y difusión de los resultados. La investigación se llevó a cabo bajo las recomendaciones de la Declaración de Helsinki y la de los derechos humanos. Los participantes fueron informados del estudio y tuvieron que firmar una hoja de consentimiento.

\section{Procedimiento}

Para conocer la percepción del staff técnico sobre la herramienta tecnológica que facilita el almacenamiento e intercambio de información, previamente se elaboró una herramienta tecnológica acorde a las necesidades del equipo, y fue aplicada con el equipo durante el transcurso de una temporada de competición (siete meses).

La herramienta tecnológica consta de una página web y su aplicación con acceso directo a la información desde dispositivos móviles. Dicha herramienta se elaboró con el objetivo de optimizar el almacenamiento e intercambio de información entre el cuerpo técnico y las deportistas. La página web se creó de manera que sólo los usuarios autorizados pudieran ver la información volcada en la misma. La herramienta tecnológica constó de los apartados que aparecen recogidos en la Figura 1.

\begin{tabular}{|l|l|}
\hline \multicolumn{1}{|c|}{$\begin{array}{c}\text { Apartados de la } \\
\text { herramienta tecnológica }\end{array}$} & \multicolumn{1}{|c|}{ Información recogida } \\
\hline Inicio & $\begin{array}{l}\text { Información sobre el club deportivo en cuestión (descripción, } \\
\text { localización, historia, patrocinadores, etc.). }\end{array}$ \\
\hline Competición & $\begin{array}{l}\text { Calendario detallado e información de la competición } \\
\text { (localización, fecha, horario). }\end{array}$ \\
\hline Eventos & Calendario visible donde se indican los eventos del equipo. \\
\hline Pretemporada & Trabajo y encuentros realizados durante la pretemporada. \\
\hline $\begin{array}{l}\text { Plan semanal-pista y plan } \\
\text { semanal-físico }\end{array}$ & $\begin{array}{l}\text { Calendario general de la semana sobre el trabajo que se va a } \\
\text { realizar con sus horarios correspondientes. }\end{array}$ \\
\hline
\end{tabular}




\begin{tabular}{|l|l|}
\hline $\begin{array}{l}\text { Trabajo físico (sala de } \\
\text { musculación) }\end{array}$ & $\begin{array}{l}\text { Trabajo detallado de cada sesión física en sala de musculación } \\
\text { (ejercicios, series y repeticiones), con una sección donde pueden } \\
\text { observar los ejercicios mediante imágenes y videos explicativos, } \\
\text { y otra para contactar con el equipo técnico. }\end{array}$ \\
\hline Pesaje & $\begin{array}{l}\text { Información volcada semanalmente sobre su índice de masa } \\
\text { corporal, el \% de grasa, el \% de agua, la masa muscular, la } \\
\text { complexión física y el metabolismo basal. }\end{array}$ \\
\hline $\begin{array}{l}\text { Estadísticas y videos del } \\
\text { equipo }\end{array}$ & $\begin{array}{l}\text { Información general y específica por jugadora del } \\
\text { comportamiento y rendimiento en juego, junto con el video de } \\
\text { cada partido. }\end{array}$ \\
\hline Informes rivales & $\begin{array}{l}\text { Informe del modelo de juego del rival y plan de partido, junto } \\
\text { con el video de juego del rival. }\end{array}$ \\
\hline Viajes & Planning de viaje (horarios, alojamientos, vestimenta, etc.). \\
\hline Normativa interna & Información sobre la normativa interna del club. \\
\hline Enlaces de interés & $\begin{array}{l}\text { Enlaces con información de distinto índole relevante para el } \\
\text { equipo. }\end{array}$ \\
\hline
\end{tabular}

Figura 1. Apartados de los que se compone la herramienta tecnológica.

Una vez que el cuerpo técnico conoció la herramienta, se realizó una posterior reunión con las jugadoras, para explicarles las características de la misma, su utilidad, su funcionamiento y darles acceso a través de sus cuentas de correo. La reunión terminó con la explicación sobre el procedimiento que debían realizar para tener un acceso directo, versión móvil, en sus dispositivos personales y comprobar que todo el equipo tenía acceso.

Posteriormente, se comenzó a incorporar el uso diario de dicha herramienta tecnológica, creada para almacenar e intercambiar información, durante la temporada. Al final de dicha temporada se evaluó su utilidad a través de entrevistas personales al staff técnico del equipo.

\section{Recogida de datos}

La técnica de recogida de datos empleada fue la entrevista individual y semiestructurada. Dicha entrevista se aplicó a los componentes del staff técnico de un equipo de voleibol femenino de alto nivel, al final de la temporada de competición. Se eligió este momento para que los atletas respondieran a las preguntas tras un uso completo de la herramienta tecnológica. El objetivo de las entrevistas fue conocer su percepción sobre la viabilidad, contextualización y utilidad de una herramienta tecnológica con posibilidad de acceso desde dispositivos móviles, empleada para almacenar e intercambiar información.

La entrevista constó de las siguientes preguntas: ¿Qué opinas sobre la viabilidad, contextualización y utilidad de la herramienta tecnológica empleada para el 
intercambio de información entre el cuerpo técnico y las jugadoras?; ¿Qué apartados de la herramienta tecnológica destacarías como más relevantes? Indica el/los motivos de ello ¿Qué apartados consideras menos relevantes? Indica el/ los motivos de ello; ¿Qué ventajas y desventajas concretas consideras que tiene utilizar este tipo de herramientas tecnológicas, con posibilidad de acceso desde dispositivos móviles, para el intercambio de información entre el cuerpo técnico y jugadoras? Las entrevistas fueron grabadas a través de una grabadora digital (Olympus VN-712PC).

\section{Análisis de los datos}

El análisis de los datos se realizó a través de la teoría fundamentada (Grounded Theory). Dicha teoría se deriva de la interacción entre la inducción y la deducción, y puede ofrecer una mejor comprensión de lo que ocurre, proporcionando información significativa ${ }^{23}$.

Los datos fueron analizados mediante un proceso de seis etapas ${ }^{38}$ : (a) Transcripción textual de las entrevistas, (b) Codificación abierta, en esta fase los datos fueron divididos en segmentos de información relevante, analizándolos inicialmente de forma independiente, para controlar el sesgo potencial. (c) Codificación axial, se utilizó este proceso de codificación para identificar los vínculos entre las categorías ${ }^{23}$. (d) Codificación selectiva, es el proceso de elección de una categoría para ser el núcleo, y relacionar todas las demás categorías con la central. (e) Una vez que se analizaron los datos, se realizó una revisión de la bibliografía existente para contextualizar los resultados hallados ${ }^{23,24}$. (f) Técnica de comprobación de miembros, en esta última etapa se volvió a contactar con el staff técnico para comprobar que la interpretación de los datos se ajustaba a su percepción real respecto al uso de la herramienta tecnológica. La recopilación y el análisis de los datos terminaron una vez que no se identificaron nuevas categorías, es decir, se alcanzó la saturación teórica ${ }^{23}$.

\section{RESULTADOS}

Los resultados indican que la herramienta tecnológica, con posibilidad de acceder desde dispositivos móviles, para almacenar e intercambiar información, es considerada una herramienta viable, contextualizada y útil, por parte del staff técnico de un equipo de voleibol de alto nivel. Además, nuestros resultados exponen los apartados de la herramienta más relevantes para los técnicos, y las ventajas y desventajas de la posibilidad de acceso a la información desde dispositivos móviles.

\section{Viabilidad, contextualización y utilidad de la herramienta}

Los resultados de nuestras entrevistas a los miembros del staff técnico muestran que la herramienta es considerada viable. Concretamente, exponen que es una herramienta de fácil incorporación y uso por un equipo deportivo. 
Es una herramienta que está al alcance de todos, puesto que todo el mundo tiene acceso a un ordenador, todo el mundo tiene acceso a la web... creo que todo el mundo tiene capacidad de incorporar su uso en su equipo y ponerlo en marcha, así que yo creo que es viable, siempre que se quiera hacer. (Entrevista entrenador asistente).

Además, uno de los aspectos destacado por el staff técnico es que la herramienta está elaborada de manera acorde con las necesidades reales del equipo, considerándola como una herramienta contextualizada.

Está muy contextualizado a un equipo que juega en una liga profesional, en nuestro caso, debemos de tener el mayor número de elementos que nos ayuden a controlar, desarrollar y transmitir información a todo el global del equipo. (Entrevista entrenador).

Inicialmente, la percepción del entrenador fue que la incorporación de esta herramienta para optimizar el almacenamiento e intercambio de información no iba a servir de utilidad al equipo. Sin embargo, su percepción cambió con el tiempo, indicando que ha sido muy útil para el control de información.

En un principio, quiero decir que, pensé como que no iba a tener utilidad ninguna pero, valorando en el tiempo, creo que el programa ha demostrado su utilidad a lo largo del tiempo... creo que controlar la información transmitida en la parte física, la parte de planificación, la parte de conocimiento del rival, la parte de trabajo táctico que se iba a hacer en cada partido y que se daba antes de nuestra reunión de análisis y preparación de partido, se demostraron muy importantes a lo largo del tiempo. (Entrevista entrenador).

En este sentido, el staff técnico indica que la herramienta le ha permitido organizar y estructurar bien la información, y de forma sencilla.

En mi opinión la herramienta está muy bien organizada y muy bien estructurada. Además, en un par de click las jugadoras podían tener la información sin necesidad de tener millones de correos o millones de papeles... (Entrevista entrenador asistente).

Además, el staff técnico considera que la herramienta ha facilitado el intercambio de información entre ellos y las jugadoras.

Personalmente, me ha resultado muy útil para poder dar información a las jugadoras, y que, además, el resto del cuerpo técnico pudiera ver toda esa información. (Entrevista entrenador asistente).

Por ello, creen que este tipo de herramientas puede ser de utilidad a cualquier equipo deportivo, o incluso, con las adaptaciones oportunas, a todo un club deportivo. 
Esta herramienta es tremendamente útil para nosotros, pero si lo extrapolas a otros equipos yo creo que les puede servir de utilidad, e incluso llevarlo mucho más allá de un solo equipo del club, llevarlo a todo el club o para poder compaginar varios equipos. (Entrevista entrenador asistente)

\section{Importancia de que todos los miembros de un equipo estén interconectados.}

En este sentido, en relación a la percepción del staff técnico sobre los apartados más relevantes, sus respuestas destacan como relevantes todos aquellos apartados que les han permitido estar interconectados con los propios miembros del staff técnico y con las jugadoras que componen el equipo.

Sobre todo, me parecen relevantes aquellos apartados que nos hacen que todos estemos interconectados. (Entrevista entrenador asistente).

En este sentido, destacan la planificación y el trabajo físico (sala de musculación).

...destaco donde se subía la planificación semanal y todos podíamos ver que teníamos que hacer cada semana y el apartado donde yo podía subir las sesiones del trabajo físico y las jugadoras las podían tener. (Entrevista entrenador asistente).

Además, el apartado de estadísticas y videos del equipo, e informes rivales, no sólo facilitaban el intercambio de información, sino que facilitaba la labor de los técnicos en las reuniones pre-partido. Esto es debido a que las jugadoras asistían a las reuniones técnicas con toda la información de los rivales revisada previamente, ayudando a optimizar el tiempo de dichas reuniones.

...poder entregarles antes de la preparación de cada partido una información sobre el equipo contrario...eso facilitaba enormemente la visualización y los análisis de los rivales, para posteriormente realizar la asignación de tareas en cada uno de los partidos. (Entrevista entrenador).

A pesar de que exponen que todos los apartados fueron importantes, el staff técnico indica que algunos de ellos fueron menos útiles, debido a que no se utilizaron con tanta frecuencia para consultar datos, como fueron el apartado de pesaje o el de viajes.

Creo que menos útil... cuando estas en el deporte de elite, no creo que haya nada menos útil, creo que todas las informaciones eran importantes, pero podemos decir que el control de peso o control alimentario, pues puede ser una de las parcelas en las que menos hemos trabajado. (Entrevista entrenador).

Pues menos útil... difícil que te lo diga...quizás el de los planes de viaje ha estado infrautilizado. (Entrevista entrenador asistente). 


\section{Ventajas e inconvenientes de la posibilidad de acceso desde dispositivos móviles.}

Los miembros del staff técnico de un equipo de alto nivel destacan como ventajas de la posibilidad de acceder a la información desde dispositivos móviles, que es cómodo, y puedes acceder desde cualquier sitio y en cualquier momento.

Pues evidentemente, que puedes acceder a la información en el momento que tú quieras. Hay jugadoras que trabajan, hay jugadoras que estudian y que cuentan con poco tiempo o que no se pueden poner delante de un ordenador, bien porque están en la universidad o porque están en su trabajo, y a través de un dispositivo móvil, pues pueden hacerlo perfectamente. (Entrevista entrenador).

Sin embargo, destacan algunos aspectos negativos o desventajas a tener en cuenta en el uso de este tipo de herramientas tecnológicas, con la posibilidad de acceder mediante dispositivos móviles. Concretamente, el staff técnico indica que pueden perder el contacto personal con el equipo.

...puedes llegar a caer en un no contacto con la plantilla, porque crees que ya le has dado toda la información, y te olvidas del contacto personal, que enriquece un montón. (Entrevista entrenador).

\section{DISCUSIÓN}

El objetivo del presente estudio fue conocer la percepción del staff técnico de un equipo de voleibol de alto nivel de la viabilidad, contextualización y utilidad, de una herramienta tecnológica, con posibilidad de acceso desde dispositivos móviles, empleada para almacenar e intercambiar información.

Nuestros resultados muestran que la incorporación de este tipo de herramientas tecnológicas, según la percepción del staff técnico de un equipo de voleibol de alto nivel, es viable, exponiendo que es una herramienta de fácil incorporación y uso. En este sentido, el uso de dicha herramienta se ha incorporado con facilidad, ya que actualmente vivimos en una sociedad informatizada ${ }^{25}$, y todos los componentes del equipo tenían unos conocimientos básicos de informática, con experiencia en el acceso y navegación web. Además, el hecho de posibilitar el acceso desde dispositivos móviles ${ }^{26}$, ha hecho muy sencilla su aplicación.

El staff técnico considera, tras el uso de la herramienta durante una temporada completa, que la misma es contextualizada y acorde a sus necesidades reales. Esto es posible ya que la herramienta se ha creado atendiendo a las necesidades que surgen en un equipo de voleibol de alto nivel. En este sentido, es relevante que todas las herramientas tecnológicas elaboradas para facilitar la vida diaria de las personas se creen atendiendo a las necesidades reales de los colectivos a los que se dirigen ${ }^{27}$. 
Nuestros resultados exponen que, inicialmente, la percepción del entrenador, sobre la incorporación de esta herramienta para optimizar el almacenamiento e intercambio de información, era que no iba a servir de utilidad al equipo. Sin embargo, su percepción cambió con el tiempo, indicando que ha sido muy útil para el control de información, permitiendo organizarla y estructurarla de forma sencilla. Además, exponen que ha facilitado el intercambio de información entre los miembros del equipo. En este sentido, son diversos los estudios que muestran que para que una herramienta tecnológica sea de utilidad, tiene que facilitar el trabajo de los usuarios ${ }^{28}$. Unido a ello, la incorporación de esta herramienta ayuda al equipo técnico a una mejor gestión e intercambio de información, debido a que es una herramienta que permite la transmisión de la misma de forma clara, significativa, sistemática y visual ${ }^{29}$.

En relación a los apartados de los que se compone la herramienta tecnológica, el staff técnico indica como más relevantes todos aquellos apartados que les han permitido estar interconectados con los propios miembros del staff técnico y con las jugadoras que componen el equipo, facilitando así, la comunicación entre los mismos, uno de los beneficios que nos pueden ofrecer las nuevas tecnologías ${ }^{30}$.

Los apartados destacados fueron: la planificación, debido a que permitía al cuerpo técnico indicar a sus jugadoras la organización de toda la semana, siendo una de las funciones más importantes del entrenador, para desarrollar adecuadamente un proyecto deportivo ${ }^{31}$; el trabajo físico (sala de musculación), donde la preparadora física podía subir toda la información con imágenes y videos explicativos, facilitando que las jugadoras pudieran trabajar sin necesidad de que ella estuviera presente, siendo una de las parcelas de trabajo imprescindibles en el alto nivel ${ }^{32}$; y las estadísticas y videos del equipo, e informes rivales, debido a que facilitaba la labor de los técnicos en las reuniones pre-partido, ya que las jugadoras asistían a las reuniones técnicas con toda la información de los rivales revisada, optimizando el tiempo de las mismas, otra de las actividades relevantes en un equipo de alto nivel ${ }^{33}$.

En un equipo de alto nivel se gestiona un gran volumen de información, y en este sentido, la herramienta tecnológica empleada sirve de ayuda en su almacenamiento e intercambio, favoreciendo la labor del staff técnico. Sin embargo, nuestros resultados exponen que los técnicos perciben algunos apartados de la herramienta como menos relevantes que el resto, se trata del pesaje o el apartado de viajes, debido a que no se utilizaron con tanta frecuencia para consultar datos. Esto puede ocurrir porque la información que los técnicos transmitían a sus deportistas mediante estos dos apartados, era aportada en otras situaciones (durante el propio pesaje o en los entrenamientos). De tal manera que, si los deportistas pueden acceder a dicha información por otros medios, la herramienta tecnológica pierde utilidad al no solventar ninguna necesidad informativa novedosa $^{27}$.

En relación a la percepción del staff técnico sobre la posibilidad de acceso a la información desde dispositivos móviles, existen beneficios e inconvenientes. En 
relación a los beneficios transmitidos por los técnicos, destacamos que es cómodo, y permite el acceso desde cualquier sitio y en cualquier momento. Concretamente, en el contexto del deporte se tienen deportistas que cuentan con poco tiempo para ponerse delante de un ordenador y acceder a la información subida a la herramienta tecnológica, bien por trabajo o estudios. Sin embargo, a través de un dispositivo móvil con acceso a internet, pueden hacerlo perfectamente ${ }^{34}$, accediendo a la información de forma inmediata ${ }^{18}$.

Sin embargo, destacan algunos aspectos negativos o desventajas a tener en cuenta en el uso de este tipo de herramientas tecnológicas, con la posibilidad de acceder mediante dispositivos móviles. Concretamente, el staff técnico indica que se puede perder el contacto personal con el equipo. En este sentido, diversos autores indican la importancia de un uso adecuado de las nuevas tecnologías para prevenir una posible dependencia ${ }^{35,36}$ o la desconexión que pueden crear con el mundo real ${ }^{37}$.

Para concluir, a pesar de ser conscientes de que el uso de las nuevas tecnologías debe hacerse de forma responsable para evitar adicciones ${ }^{38}$, sin lugar a dudas, es una de las herramientas de trabajo que podemos incorporar en el ámbito del entrenamiento para facilitar la labor diaria de técnicos y deportistas ${ }^{39}$. Por ello, este trabajo destaca, a través de la percepción del staff técnico de un equipo de voleibol de alto nivel, que la incorporación de una herramienta tecnológica con posibilidad de acceso desde dispositivos móviles, para el almacenamiento e intercambio de información, es viable, contextualizado y útil para la gestión de información, permitiendo organizarla y estructurarla de forma sencilla. Además, nuestros resultados exponen que ha facilitado el intercambio de información entre los miembros del equipo y que la posibilidad de acceso mediante un dispositivo móvil tiene grandes ventajas. Por ello, sugerimos a los equipos deportivos que manejan, gestionan e intercambian grandes cantidades de información, que incorporen dichas tecnologías para facilitar su trabajo diario.

\section{REFERENCIAS BIBLIOGRÁFICAS}

1. Baca A, Dabnichki P, Heller M, Kornfeind P. Ubiquitous computing in sports: A review and analysis. Journal of Sports Sciences. 2009; 27(12):1335-1346. https://doi. org/10.1080/02640410903277427.

2. Sharp B. (1996). The use of computers in sports science. British journal of educational technology.1996; 27(1):5-32. https://doi.org/10.1111/j.1467-8535.1996.tb00140.

3. Liebermann DG, Katz L, Hughes MD, Bartlett RM, McClements J, Franks IM. Advances in the application of information technology to sport performance. Journal of Sports Sciences. 2002; 20(10):755-769. https://doi.org/10.1080/0264041023206756 11.Ç

4. Buscà B, Quintana M, Padullés JM. High-speed cameras in sport and exercise: Practical applications in sports training and performance analysis. Aloma: revista de psicologia, ciències de l'educació i de l'esport Blanquerna. 2016; 34(2):11-24. 
5. Donnelly JE. Using microcomputers in physical education and the sport sciences. Champaign: HumanKinetics Publishers; 1987.

6. Haake SJ. The impact of technology on sporting performance in Olympic sports. Journal of Sports Sciences. 2009; 27(13):1421-1431. https://doi. org/10.1080/02640410903062019.

7. Vale AP, Martínez CIP, Pedré D. La informática y las comunicaciones en el deporte: una revisión bibliográfica. CD de Monografías. 2012: 1-12.

8. Ibanez SJ, Pérez MA, Macías M. Software for the basketball player's evaluation in real time. International Journal of Performance Analysis in Sport. 2003; 3(2):145-155. https://doi.org/10.1080/24748668.2003.11868285.

9. Smith T, Hammond J, Gilleard W. The use of performance analysis technology to monitor the coaching environment in soccer. International Journal of Performance Analysis in Sport. 2005; 5(3):126-138. https://doi.org/10.1080/24748668.2005.11868 343.

10. Morante JC, Villa JG, García ZJ. Aplicación de nuevas tecnologías como herramienta de ayuda para los entrenadores de voleibol: el software VoleyTrain. Kronos. 2002; 1(1-2):1-5.

11. Qi C, Wei H, Shu-ming X, Zhi-bin T, Hong-wei C, Yan-qing D, Hong-fan S. Development of Wireless Sensor Network for Data Collection and Footwork Training. Procedia Engineering. (2012); 34:520-525. https://doi.org/10.1016/j.proeng.2012.04.089.

12. Strohrmann C, Harms H, Kappeler-Setz C, Troster G. Monitoring kinematic changes with fatigue in running using body-worn sensors. IEEE Transactions on Information Technology in Biomedicine. 2012; 16(5):983-990. https://doi.org/10.1109/ TITB.2012.2201950.

13. James DA, Leadbetter RI, Neeli MR, Burkett BJ, Thiel DV, Lee JB. An integrated swimming monitoring system for the biomechanical analysis of swimming strokes. Sports Technology. 2011; 4(3-4):141-150. https://doi.org/10.1080/19346182.2012.725410.

14. Chambers R, Gabbett TJ, Cole MH, Beard A. The use of wearable microsensors to quantify sport-specific movements. Sports Medicine. 2015; 45(7): 1065-1081. https:// doi.org/10.1007/s40279-015-0332-9.

15. Sampaio J, Maçãs V. Measuring tactical behaviour in football. International Journal of Sports Medicine. 2012; 33(05):395-401. https://doi.org/10.1055/s-0031-1301320

16. Sanz D, Terroba A. New technologies applied to tactical analysis in tennis. Coaching \& Sport Science Review. 2012; 56:22-24.

17. Garita-Araya RA. Tecnología Móvil: desarrollo de sistemas y aplicaciones para las Unidades de Información. Revista e-Ciencias de la Información. 2013; 3(2):1-14. https://doi.org/10.15517/eci.v3i2.10654.

18. Enriquez JG, Casas SI. Usabilidad en aplicaciones móviles. Informes CientíficosTécnicos UNPA. 2014; 5(2):25-47. 
19. Padmasekara G. Fitness apps, a Valid Alternative to the Gym: a pilot study. Journal of Mobile Technology in Medicine. 2014; 3(1):37-45. https://doi.org/10.7309/ jmtm.3.1.6.

20. Eskofier B, Hartmann E, Kühner P, Griffin J, Schlarb H, Schmitt M, Hornegger J. Real time surveying and monitoring of athletes using mobile phones and GPS. International Journal of Computer Science in Sport. 2008; 7(1):18-27.

21. Balsalobre-Fernández $C$, Glaister M, Lockey RA. The validity and reliability of an iPhone app for measuring vertical jump performance. Journal of Sports Sciences. 2015; 33(15):1574-1579. https://doi.org/10.1080/02640414.2014.996184.

22. Bort-Roig J, Gilson ND, Puig-Ribera A, Contreras RS, Trost SG. Measuring and influencing physical activity with smartphone technology: a systematic review. Sports Medicine. 2014; 44(5):671-686. https://doi.org/10.1007/s40279-014-0142-5.

23. Strauss A, Corbin J. Basics of qualitative research techniques: Techniques and Procedures for Developing Grounded Theory. Thousand Oaks, CA: Sage; 1998.

24. Holt NL, Dunn JG. Toward a grounded theory of the psychosocial competencies and environmental conditions associated with soccer success. Journal of Applied Sport Psychology. 2004; 16(3):199-219. https://doi.org/10.1080/10413200490437949.

25. Gilbert JK. Educación tecnológica: una nueva asignatura en todo el mundo. Enseñanza de las Ciencias: Revista de Investigación y Experiencias Didácticas. 1995; 13(1):15-24.

26. Tognazzi Drake A. Las transformaciones de los contenidos audiovisuales y la influencia de los dispositivos móviles en el nuevo escenario transmedia. Anàlisi: quaderns de comunicació i cultura. 2012: 81-95. https://doi.org/10.7238/a.v0iMonografico.1503.

27. Riguet-Riot C, Carter S, James DA. Programmed Innovation in team sport using needs driven innovation. Procedia Engineering. 2014; 72:817-822. https://doi. org/10.1016/j.proeng.2014.06.139.

28. Davis FD, Bagozzi RP, Warshaw PR. User acceptance of computer technology: a comparison of two theoretical models. Management Science. 1989; 35(8):982-1003. https://doi.org/10.1287/mnsc.35.8.982.

29. Oña A, Martínez M, Moreno F. Descripción de un sistema informatizado de procesamiento automático para la optimización del rendimiento deportivo basado en el control de la información. European Journal of Human Movement. 1994; 1:57-69.

30. García FG. De la convergencia tecnológica a la convergencia comunicativa en la educación y el progreso. Revista ICONO14 Revista Científica de Comunicación y Tecnologías emergentes. 2006; 4(1):1-19. https://doi.org/10.7195/ri14.v4i1.394.

31. Feu S, Ibáñez SJ, Gozalo M. Propiedades psicométricas de los cuestionarios EDD y EPD para evaluar el estilo de planificación y decisión de los entrenadores. Revista de Psicología del Deporte. 2007; 16(2):185-199. 
32. Marqués MC, Van Den Tillaar R, Vescovi JD, González-Badillo JJ. Changes in strength and power performance in elite senior female professional volleyball players during the in-season: a case study. The Journal of Strength \& Conditioning Research. 2008; 22(4):1147-1155. https://doi.org/10.1519/JSC.0b013e31816a42d0.

33. Sarmento H, Bradley P, Travassos B. The transition from match analysis to intervention: Optimising the coaching process in elite futsal. International Journal of Performance Analysis in Sport. 2015; 15(2):471-488. https://doi.org/10.1080/24748668.20 15.11868807.

34. Niño JIN, Fernández BF. Comunicación, Salud y Tecnología: mHealth. Revista de Comunicación y Salud. 2015; 5:144-153.

35. Encinas FJL, González SMV. Menores y nuevas tecnologías: conductas indicadoras de posible problema de adicción. Psicothema. 2010; 22(2):180-188.

36. Fuster H, Chamarro A, Oberst U. Fear of Missing Out, online social networking and mobile phone addiction: A latent profile approach. Aloma: Revista de Psicologia, Ciències de l'Educació i de l'Esport. 2017; 35(1):23-30.

37. Echeburúa E, De Corral P. Adicción a las nuevas tecnologías ya las redes sociales en jóvenes: un nuevo reto. Adicciones. 2010; 22(2):91-95. https://doi.org/10.20882/ adicciones.196.

38. Arias O, Gallego VG, Rodríguez MJ, Del pozo MA. Adicción a las nuevas tecnologías. Psicología de las Adicciones. 2012; 1: 2-6.

39. Morante JC, Villa JG. Valoración técnico-táctica y control del entrenamiento a través de programas informáticos. Rendimiento Deportivo.com. 2002; 1. http://www. Rendimientodeportivo.com. [Consulta: 26/02/18].

Referencias totales citadas: 39

Referencias citadas correspondientes a la Rev Ib CC Act Fis Dep: 0 